\title{
Analysis of the impact of COVID-19 on some medical institutions in a certain region
}

\author{
Junliang $\mathrm{Li}^{1}$, Junying $\mathrm{Li}^{2}$, Huiqin Zhang ${ }^{3}$, Xiuli $\mathrm{Bai}^{4}$, Lingfeng Wang*1 \\ ${ }^{1}$ The Third Affiliated Hospital of Inner Mongolia Medical University (Baogang Hospital), Baotou, Inner Mongolia, China \\ ${ }^{2}$ Baotou Eighth Hospital, Baotou, Inner Mongolia, China \\ ${ }^{3}$ Baotou Health Statistics Information Center, Baotou, Inner Mongolia, China \\ ${ }^{4}$ Baotou Central Hospital, Baotou, Inner Mongolia, China
}

Received: October 18, 2020

DOI: $10.5430 /$ dcc.v7n $4 p 4$
Accepted: December 30, 2020 Online Published: January 4, 2021

URL: https://doi.org/10.5430/dcc.v7n4p4

\begin{abstract}
Objective: This study is designed to analyze the impact of COVID-19 on hospitals in a certain region through the statistical comparison of outpatient, emergency and inpatient data of three representative AAA hospitals in this region from January and February of 2019 and 2020.

Methods: Relevant outpatient, emergency and inpatient data of three representative AAA hospitals in a certain region from January and February of 2019 and 2020 were collected, and the statistical analysis was conducted by using EXCEL 2019 and SPSS20.0.

Results: In January and February of 2020, the number of the outpatient, the emergency, the inpatient and the inpatient surgical interventions in the three hospitals was significantly decreased in comparison with the same period in 2019, and the change of the emergency dispatching amount was relatively small. The mortality of the inpatient in January and February in 2020 was about $2.82 \%$, and that in the same period in 2019 was about $1.51 \%$, with an increase of about $1.31 \%$, which presented a statistically significant difference $\left(\chi^{2}=348.422 ; p=.000\right)$.

Conclusions: The outbreak of COVID-19 had a great impact on the normal operation of the hospital.
\end{abstract}

Key Words: COVID-19, Hospital, Impact

\section{INTRODUCTION}

Since the outbreak of Corona Virus Disease 2019 (COVID2019), the Party Central Committee and the State Council have put a new premium on it and taken a strong action to prevent the spread of the epidemic. However, as a sudden public health crisis, the outbreak of the epidemic has a huge impact on the life health and the economic society. As one of the main battlefields for fighting against the epidemic of
COVID-2019, hospitals were severely affected by the outbreak of the epidemic in terms of the normal operation. How to prevent or relieve from the "secondary disaster" caused by the epidemic? This study is designed to analyze the impact of COVID-19 on hospitals in this region through the statistical comparison of outpatient, emergency and inpatient data of three representative AAA hospitals in this region from January and February of 2019 and 2020.

\footnotetext{
${ }^{*}$ Correspondence: Lingfeng Wang; Email: wlf7413@vip.sina.com; Address: The Third Affiliated Hospital of Inner Mongolia Medical University (Baogang Hospital), Baotou, Inner Mongolia 014010, China.
} 


\section{DATA AND METHODS}

The data source was true and credible as the data were collected from the Department of Statistics from each of three AAA hospitals. EXCEL 2019 and SPSS20.0 were used to make a statistical analysis of the above data from January and February of 2019 and 2020.

\section{Results}

\subsection{The number of the outpatient}

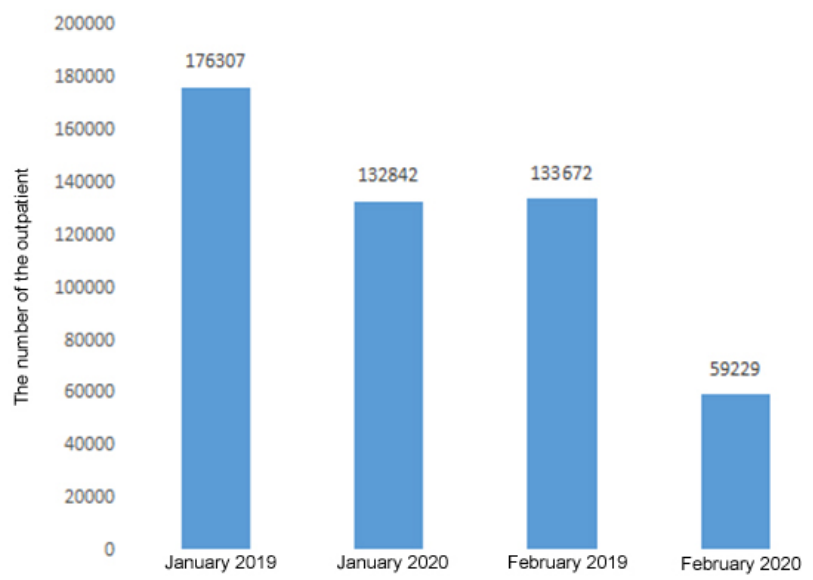

Figure 1. The number of the outpatient in January and February of 2019 and 2020

\subsection{The number of the emergency}

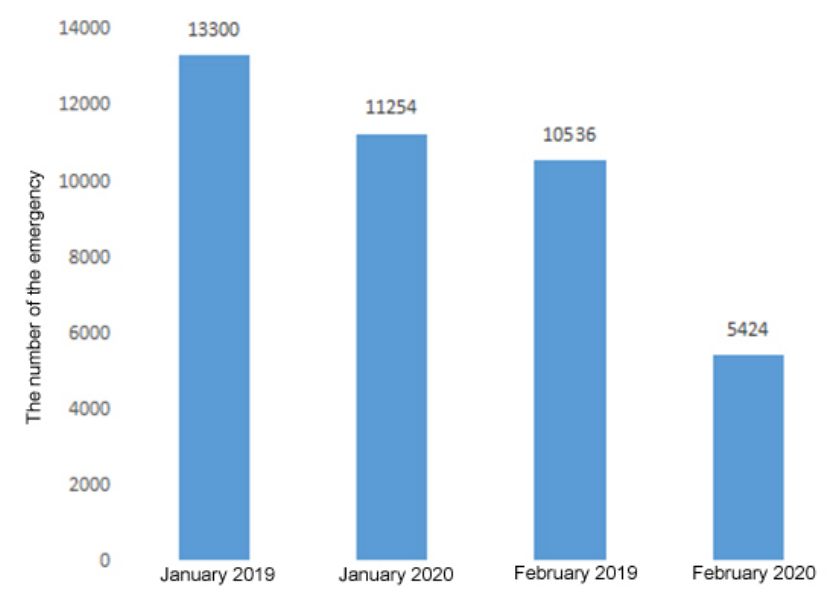

Figure 2. The number of the emergency in January and February of 2019 and 2020

\subsection{Emergency dispatching amount}

1600

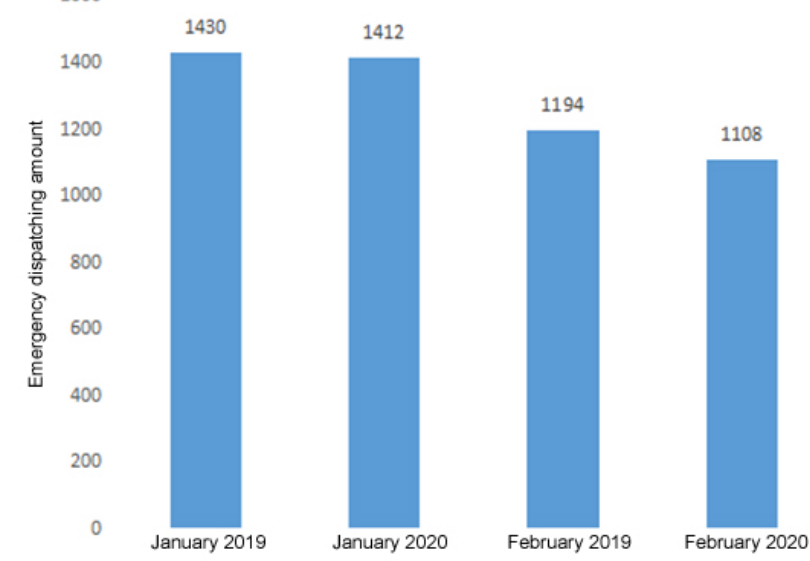

Figure 3. Emergency dispatching amount

\subsection{The number of the inpatient}

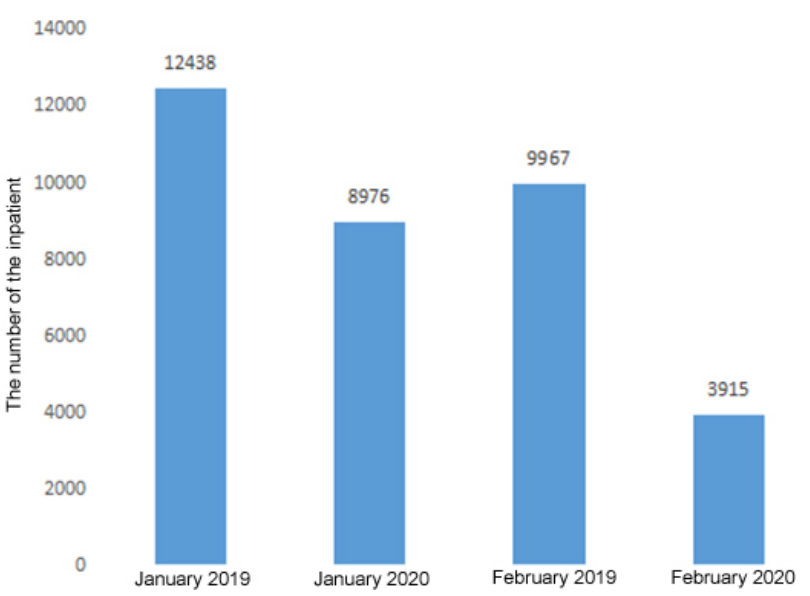

Figure 4. The number of the inpatient in January and February of 2019 and 2020

\subsection{The number of the inpatient surgical interventions}
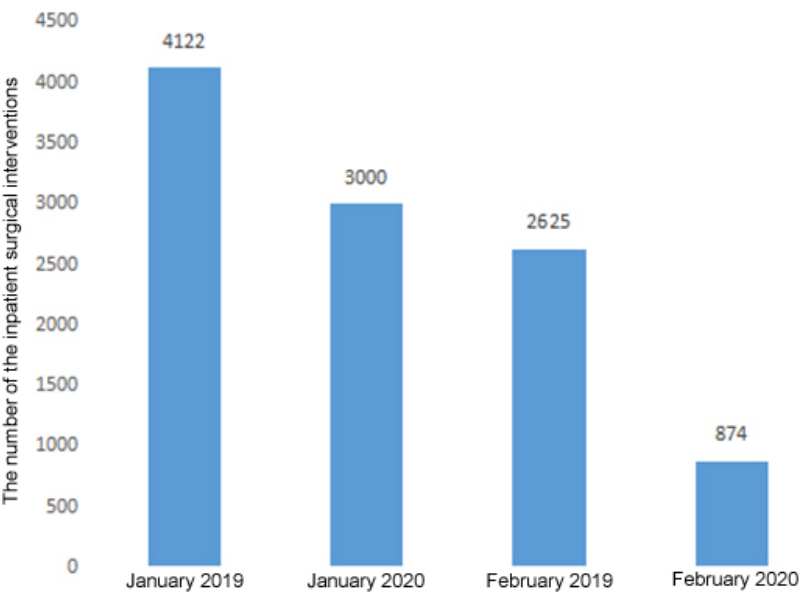

Figure 5. The number of the inpatient surgical interventions in January and February of 2019 and 2020 


\subsection{The morality of the inpatient}

Table 1. The morality of the inpatient in January and February of 2019 and 2020

\begin{tabular}{lllll}
\hline Item & January of $\mathbf{2 0 1 9}$ & January of 2020 & February of 2019 & February of 2020 \\
\hline The number of inpatient death & 197 & 195 & 142 & 169 \\
The number of the inpatient & 12,438 & 8,976 & 9,967 & 3,915 \\
Proportion & $1.58 \%$ & $2.17 \%$ & $1.42 \%$ & $4.32 \%$ \\
\hline
\end{tabular}

\subsection{Types of diseases causing inpatient death}

Table 2. Types and proportion of diseases causing inpatient death in January and February of 2019 and 2020

\begin{tabular}{|c|c|c|c|c|c|c|}
\hline $\begin{array}{l}\text { Serial } \\
\text { Number }\end{array}$ & $\begin{array}{l}\text { Types of diseases causing inpatient } \\
\text { death in January and February of } 2019\end{array}$ & $\mathbf{n}$ & Proportion & $\begin{array}{l}\text { Types of diseases causing inpatient } \\
\text { death in January and February of } 2020\end{array}$ & $\mathbf{n}$ & Proportion \\
\hline 1 & Pulmonary Infection & 36 & $10.62 \%$ & Pulmonary Infection & 68 & $18.68 \%$ \\
\hline 2 & Malignant Tumor & 33 & $9.73 \%$ & Malignant Tumor & 47 & $12.91 \%$ \\
\hline 3 & Cerebral Hemorrhage & 33 & $9.73 \%$ & Cerebral Hemorrhage & 35 & $9.62 \%$ \\
\hline 4 & Acute outbreak of COPD & 24 & $7.08 \%$ & Cerebral Ischemic Stroke & 23 & $6.32 \%$ \\
\hline 5 & Myocardial Infarction & 14 & $4.13 \%$ & Myocardial Infarction & 12 & $3.30 \%$ \\
\hline 6 & Acute outbreak of Chronic bronchitis & 11 & $3.24 \%$ & Acute outbreak of COPD & 10 & $2.75 \%$ \\
\hline 7 & Left Heart Failure & 6 & $1.77 \%$ & Grade 3 Cardiac Function & 5 & $1.37 \%$ \\
\hline 8 & Grade 3 Cardiac Function & 6 & $1.77 \%$ & Pneumosilicosis & 3 & $0.82 \%$ \\
\hline 9 & Head Injury & 4 & $1.18 \%$ & Acute Pulmonary Embolism & 3 & $0.82 \%$ \\
\hline \multirow[t]{5}{*}{10} & Chronic Renal Failure & 3 & $0.88 \%$ & Cardiac Standstill & 3 & $0.82 \%$ \\
\hline & & & & Circulatory Failure & 3 & $0.82 \%$ \\
\hline & & & & Alimentary Tract Hemorrhage & 3 & $0.82 \%$ \\
\hline & & & & Intestinal Obstruction & 3 & $0.82 \%$ \\
\hline & & & & Spinal Angioma & 2 & $0.55 \%$ \\
\hline
\end{tabular}

\section{Discussion}

According to the statistics from three comprehensive AAA hospitals, the number of the outpatient/emergency and the number of the inpatient in the three hospitals in this region in 2018 accounted for $67.38 \%$ and $63.41 \%$, respectively, of the total number of diagnosis and treatment of all the hospitals in this region. These three hospitals can reflect the diagnosis and treatment condition of medical institutions in this region. From the statistics mentioned above, the number of the outpatient, the emergency, the inpatient and the inpatient surgical interventions was significantly decreased in January and February of 2020 in comparison with the same period in 2019, with small changes in the number of the emergency dispatching.

The morality of the inpatient of the three hospitals in January and February of 2020 was above $2.82 \%$, and that in the same period in 2019 was around $1.51 \%$, with an increase of about $1.31 \%$. The difference was of statistical significance $\left(\chi^{2}=\right.$ $348.422 ; p=.000)$. The morality of the inpatient of the three hospitals in January of 2020 was above $2.17 \%$, and that in the same period in 2019 was around $1.58 \%$, with an increase of about $0.59 \%$. The difference was of statistical significance $\left(\chi^{2}=9.681 ; p=.002\right)$. The morality of the inpatient of the three hospitals in February of 2020 was above $4.32 \%$, and that in the same period in 2019 was around $1.42 \%$, with an increase of about $2.90 \%$. The difference was of statistical significance $\left(\chi^{2}=101.406 ; p=.000\right)$.

The number of inpatient death caused by top 10 types of diseases in the three hospitals in January and February of 2020 accounted for $60.44 \%$ of the total number of inpatient death in the same period, and the number of inpatient death caused by top 5 types of diseases possessed $50.82 \%$. The number of inpatient death caused by top 10 types of diseases in the three hospitals in January and February of 2019 accounted for $50.15 \%$ of the total number of inpatient death in the same period, and the number of inpatient death caused by top 5 types of diseases possessed $41.30 \%$. There was no change and difference in the order of top three types of diseases in January and February of 2019 and $2020\left(\chi^{2}=\right.$ $3.333 ; p=.189)$. In comparison with the same period in 
2019 , there were 58 cases of death caused by cerebral disease, with an increase of 25 cases; 84 cases of death caused by pulmonary disease, with an increase of 13 cases; 47 cases of death caused by tumors, with an increase of 14 cases; and 23 cases of death caused by cardiovascular diseases, with a decrease of 3 cases. Cerebral infarction, which didn't rank the top 10 types of diseases causing inpatient death in 2019, rose to the fourth type of disease causing inpatient death in 2020. The above-mentioned results were probably caused by the following factors: 1) During the outbreak of the epidemic, patients subjectively avoided going to hospital for fear of infection and chose home care. They had to go to hospital only when the physical condition got worse. 2) It was inconvenient to seek medical advice as the hospitals and communities enhanced the management of prevention and control. 3) The common people were so insufficient in the knowledge of health care that it affected the pre-judgment of the disease. 4) The community health management was not complete so that the probability of the occurrence of disease was accordingly increased. 5) During the outbreak of the epidemic, the medical personnel undertook more pressures from the prevention and control of COVID-2019 and weakened the administration of common diseases. 6) No establishment of designated hospitals for treating non-febrile patients affected their attendance to hospital.

WHO identified the pathogen causing the outbreak of the epidemic as Corona Virus Disease 2019 (COVID-2019). On January $30^{t h}$, WHO announced that COVID-2019 was classified as public health emergency of international concern. ${ }^{[1]}$ Public health emergency usually relates to the individual, the community (system or department) and other main social subjects, and has a widespread and worldwide effect. ${ }^{[2]}$ As public events spread quickly, they raise public concerns and become the public hot topics, causing public damage, public psychological panic and social disorder. From the perspective of medical institutions, this study proposes the following recommendations to cope with significant public health emergencies in combination with this epidemic:

(1) In light of the actual conditions, it is recommended to optimize the layout configuration of medical resources, adopt "peacetime-wartime" pattern and consolidate the construction of specialized hospitals for infectious diseases to establish "peacetime-wartime" hospitals or inpatient buildings for infectious diseases which are consistent with infectious disease management requirements. In the "peacetime," these hospitals can handle normal patients, while in the "wartime," they were transformed into hospitals or buildings for infectious disease patients in order to make full use of available beds in the "peacetime" in view of the shortage of beds in the "wartime." Particularly, it is recommended to construct conforming buildings for infectious patients in comprehensive AA hospitals and establish standardized ICUs for infectious patients in comprehensive AAA hospitals, so that the capability of emergent supply in the hospitals will be intensely strengthened.

(2) It is recommended to make a consolidation of hospitals for infectious diseases with comprehensive hospitals to set up a medical group in order to make an integrated management and guarantee the promotion of the prevention and control level and comprehensive treatment capacity. In this way, it is easy to resolve the lack of patients in the hospitals for infectious diseases in the non-epidemic period, meanwhile, comprehensive hospitals can work as a strong and firm technical support to make up for the deficiency in medical care capacity for critically ill patients in the hospitals for infectious diseases.

(3) It is recommended to strengthen the construction of infectious diseases departments in AA and AAA comprehensive hospitals. It is recommended to increase input in the public hospitals, establish standardized infectious disease departments, improve the function and the structure, enhance talent team construction and guarantee the normal operation. It is recommended to establish a new mechanism for reserve of technical talents, so that the doctors in infectious disease departments will have work to do and make a difference in the "peacetime."

(4) It is recommended to strengthen regional health planning, coordinate regional medical resources and guarantee regular patients and infectious disease patients respectively go to different designated hospitals during the outbreak of significant epidemic. It is required to not only guarantee the effective control of the epidemic, but also guarantee a safe and reliable treatment environment for non-infectious patients.

(5) It is recommended to set up a new mechanism for the normalization of the prevention and control of infectious diseases. In the past, comprehensive hospitals didn't take the reception of infectious disease patients into consideration in the aspects of planning, construction, layout and process. In these hospitals, the buildings are densely distributed, mainly in highrise buildings, with a high risk of nosocomial infections. Hospitals are needed to make a new planning or a reconstruction to complete their functions according to the requirements of screening, receiving, isolating and treating infectious disease patients. 
(6) It is recommended to deepen the reform of medical care system and complete the hierarchical medical system. It is imperative to strengthen the construction of basic medical institutions to realize basic first visit, dual referral, acute-chronic partition and coordination at different levels, and then strengthen the construc- tion of public health and service system to gradually complete people's health care system.

\section{CONFlicts OF INTEREST Disclosure}

The authors declare they have no conflicts of interest.

\section{REFERENCES}

[1] World Health Organization. Statement on the second meeting of the International Health Regulations (2005) Emergency Committee regarding the outbreak of novel corona virus (2019-nCoV) [EB/OL]. [2020-01-31]. Available from: https ://www . who.int/news-room/detail/30-01-2020-t atement-on-the-second-meeting-of-the-international -health-regulations-2005-emergency-committee-regar ding-the-outbreak-of-novel-coronavirus-2019-ncov

[2] Wang P, Liang X. Effect of professional societies on coping with public health emergency - taking COVID-2019 as an example. Administration Reform. 2020; 3: 18-23. 